\section{Fenestration of a covered metal stent during cysto- duodenostomy using argon plasma coagulation}

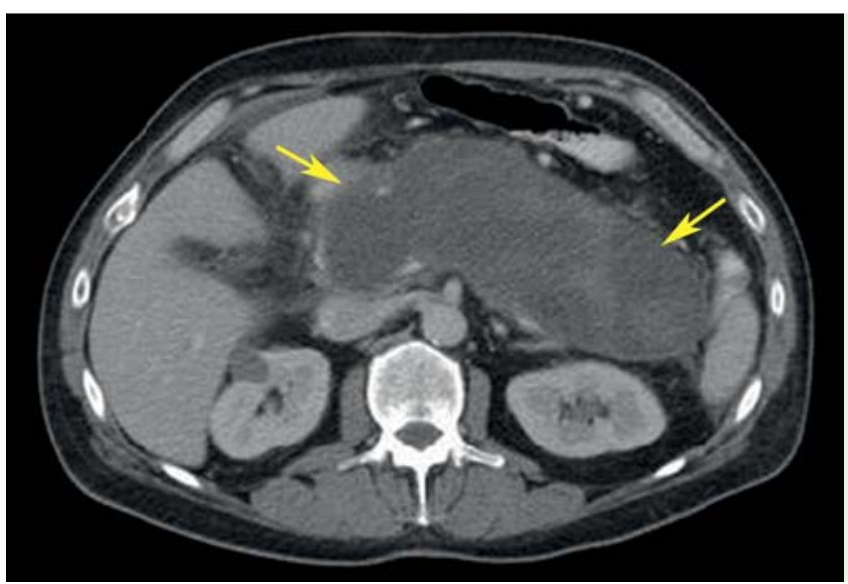

Fig. 1 Axial computed tomography (CT) scan showing a large heterogeneous peripancreatic fluid collection lying along the entire pancreas, measuring approximately $18 \times 8 \times$ $13 \mathrm{~cm}$ (yellow arrows).

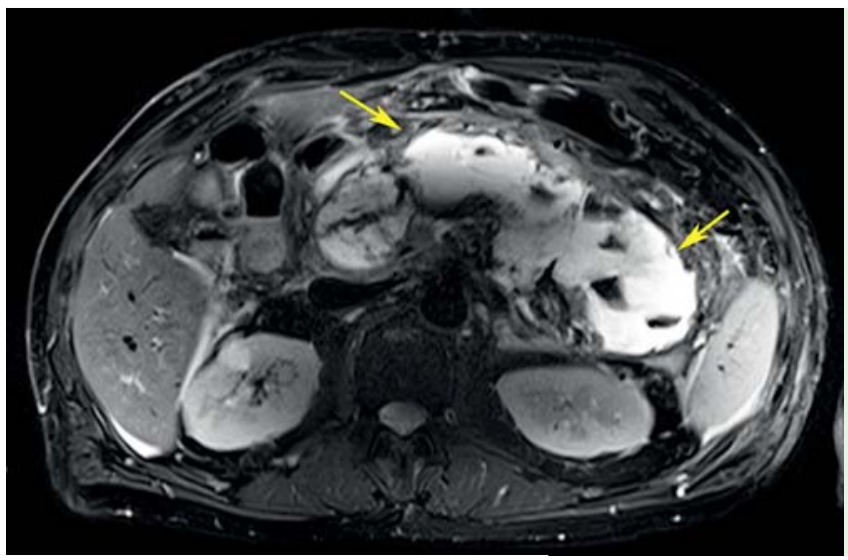

Fig. 2 T2-weighted magnetic resonance imaging (MRI) scan showing a large fluid collection, measuring approximately $18 \mathrm{~cm}$ in cross-section, with at least $70 \%$ of the fluid containing hemorrhagic and partially necrotic debris (yellow arrows).

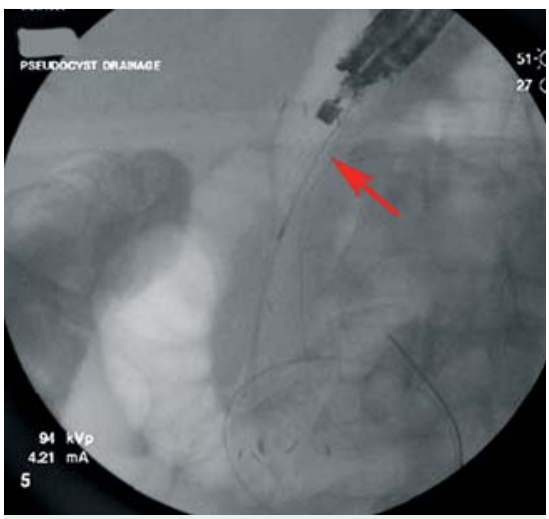

Fig. 3 Fluoroscopic image of the fully covered self-expandable metal stent (FCSEMS) placed across the cystoduodenostomy (red arrow).

The current endoscopic methods for the treatment of walled-off pancreatic necrosis (WOPN) include endoscopic ultrasound (EUS)-guided cystenterostomy with placement of a fully covered self-expandable metal stent (FCSEMS). Studies have shown that FCSEMSs designed specifically for drainage of pancreatic fluid colavailable in the USA [1]. Esophagea ate endoscopic accessories and stents. (๑ Fig. 3).
The wide-caliber stent was chosen to allow for easy drainage of necrotic debris without risk of clogging of the stent and for repeated access to the walled-off area for direct endoscopic necrosectomy, if needed. The proximal aspect of the stent was deployed within the duodenal lumen. However, because of the limited diameter of the duodenum, the flared end and shaft of the stent occupied the entire duodenal lumen, resulting in duodenal obstruction. To overcome this mechanical obstruction, argon plasma coagulation (APC) was used at 60 Watts and $1 \mathrm{~L} / \mathrm{min}$ flow to fenestrate the stent interstices ( $\bullet$ Fig. 4; $\bullet$ Video 1 ). The fenestrations provided a patent lumen through which food and the necrotic pancreatic contents could pass into the duodenum and onwards through the gastrointestinal tract.

The patient was restarted on an oral diet on the day following the procedure and was tolerating this well prior to discharge. He reported a rapid resolution of his symptoms, and repeat imaging at 6 weeks showed a significant reduction in the size of the WOPN ( $\bullet$ Fig. 5).

The use of APC for trimming metal stents has been reported in a few cases $[3,4]$. Here we report a novel use of APC to fenestrate a metal stent and tailor it to the needs of a patient with an area of WOPN and altered gastrointestinal anatomy.

\section{Video 1}

Successful fenestration of a fully covered selfexpandable metal stent (FCSEMS) during cystoduodenostomy using argon plasma coagulation (APC). FCSEMSs can also be used for the same purpose [2]. Technical success rates can be limited by the availability of appropriA 54 year-old man who had undergone prior esophagectomy and gastric pull-up was admitted with symptomatic WOPN. Abdominal imaging revealed an area of WOPN of $18 \times 13 \times 8 \mathrm{~cm}$ that was causing an extrinsic mass effect on the gastrointestinal tract ( $\bullet$ Fig. 1 and $\bullet$ Fig. 2). EUSguided drainage was performed. The altered anatomy mandated a cystoduodenostomy. A 19G needle was used to puncture the walled-off area and a 0.035-inch guidewire was coiled within the necrotic area. The tract was dilated to $10 \mathrm{~mm}$. An $18 \times 60-\mathrm{mm}$, through-the-scope FCSEMS (TaeWoong Medical, Seoul, Korea) was placed across the cystoduodenostomy
Competing interests: Mouen A. Khashab Olympus America and has received research support from Cook Medical.

Payal Saxena has received consulting fees from Boston Scientific and research support from Cook Medical.

Anthony Kalloo is a founding member, equity holder, and consultant for Apollo Endosurgery.

Vikesh K. Singh is a consultant for Abbvie, Santarus, D-Pharm, and Boston Scientific. Anne Marie Lennon is a consultant for Boston Scientific.

The remaining authors have no conflicts of interest to declare. is a consultant for Boston Scientific and 


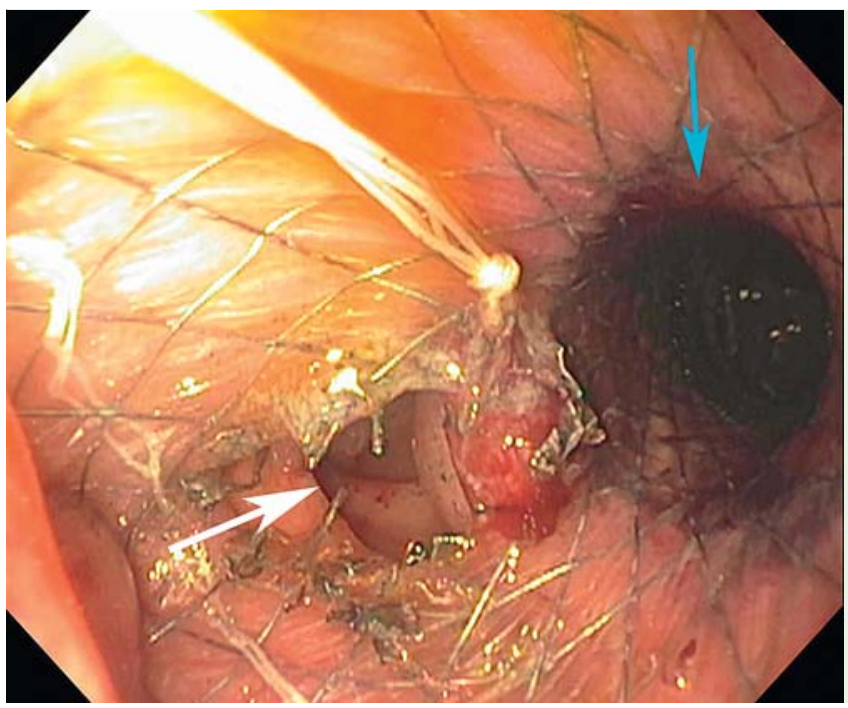

Fig.4 Endoscopic image of the fully covered self-expandable metal stent (FCSEMS) placed across the cystenterostomy (blue arrow), with the partially obstructed duodenal lumen visible through the fenestrated stent interstices (white arrow).

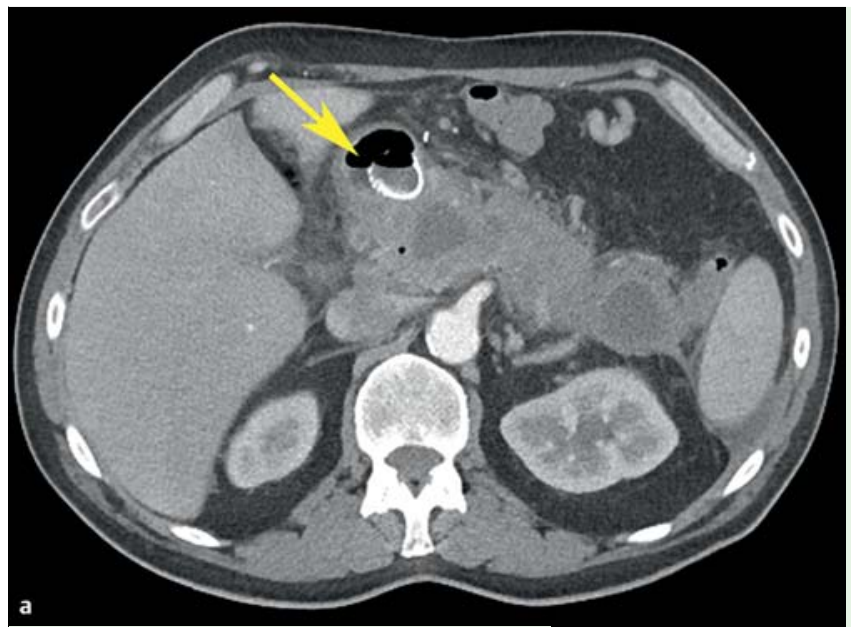

Fig. 5 Computed tomography (CT) scan images 6 weeks after fully covered, self-expandable metal stent (FCSEMS) placement showing: a (in axial view) a significant reduction in the size of the walled-off area of pancreatic necrosis (yellow arrow); b (in coronal view) the FCSEMS in position between the duodenum and the walled-off area (green arrow).

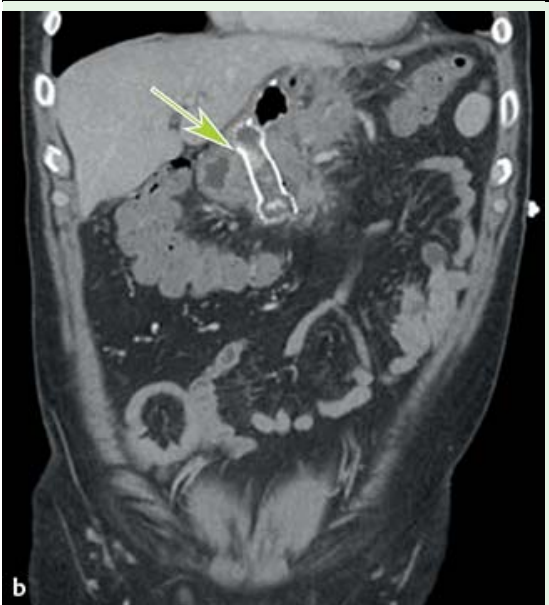

Alan H. Tieu, Payal Saxena,

Vikesh K. Singh, Anne Marie Lennon, Vivek Kumbhari, Ahmed Messallam, Mohamad El Zein, Anthony N. Kalloo, Mouen A. Khashab

Department of Medicine and Division of Gastroenterology and Hepatology, The Johns Hopkins Medical Institutions, Baltimore, Maryland, USA

\section{References}

1 Yamamoto $\mathrm{N}$, Isayama $\mathrm{H}$, Kawakami $\mathrm{H}$ et al. Preliminary report on a new, fully covered, metal stent designed for the treatment of pancreatic fluid collections. Gastrointest Endosc 2013; 77: 809-814

2 Kahaleh M. Endoscopic necrosectomy for walled-off pancreatic necrosis. Clin Endosc 2012; 45: 313-315

3 Demarquay JF, Dumas R, Peten EP et al. Argon plasma endoscopic section of biliary metallic prostheses. Endoscopy 2001; 33: 289290

4 Hamada $T$, Nakai $Y$, Isayama $H$ et al. Trimming a covered metal stent during hepaticogastrostomy by using argon plasma coagulation. Gastrointest Endosc 2013; 78: 817

Bibliography

Dol http://dx.doi.org/

10.1055/s-0034-1377363

Endoscopy 2014; 46: E512-E513

(C) Georg Thieme Verlag KG

Stuttgart · New York

ISSN 0013-726X

\section{Corresponding author}

\section{Mouen A. Khashab, MD}

Department of Therapeutic Endoscopy

Johns Hopkins Hospital

1800 Orleans St, Suite 7125 B

Baltimore

MD 21205

USA

mkhasha1@jhmi.edu 